\title{
THE POST OFFICE RESEARCH STATION
}

T HE Post Office Engineering Research Station at Dollis Hill held an open day on September 27, when several hundred visitors from the universities, industry and Government departments were able to see some of the work in progress or recently completed. Some exhibits were of exploratory work, but most were of new apparatus or systems. The contributions made by the Station to several notable developments in telecommunications since the last open day (in 1954) were reflected in the exhibits; only that to the development of electronic telephone exchanges, now being undertaken jointly with industry, was not given prominence.

Submarine telephony was represented by several diverse exhibits, including some of the equipment and techniques used for the first trans-Atlantic telephone cable which had celebrated the first anniversary of the opening of its service a day or so earlier; others were intended for future use. A length of the new light-weight deep-sea cable proposed by the Post Office was exhibited; its cross-section is shown in Fig. 1. The placing of the strength members inside the inner conductor reduces the size and cost of the eable and renders it nearly kinkless. A full-scale deep-sea trial is planned. The technique developed for jointing lengths, by swaging a stoel ferrule over the steel strands, retains at least 94 per cent of the intrinsic strength of the cable. Only one repeatered submarine cable is necessary for a system designod by the British Post Office, because the amplifier is so associated with directional filters as to be able to amplify sixty conversations in each direction simultaneously. The complete repeater is bulky and rigid, however, and its laying has hitherto presented difficulties; it cannot be passed over or around the normal laying drums. A method of laying is being developed, in which the repeater by-passes new cable-laying equipment; it will not require the ship to be stopped. It was demonstrated by a scale model in the new hydraulics laboratory, where visitors could see also the large pressure vessels and machinery for testing repeaters and their glands at pressures up to the highest reached on the ocean bed, 6 tons/sq. in. The plant for making the glands which seal the repeater against the ingress of water at such high pressures, and the portable low-voltage X-ray-

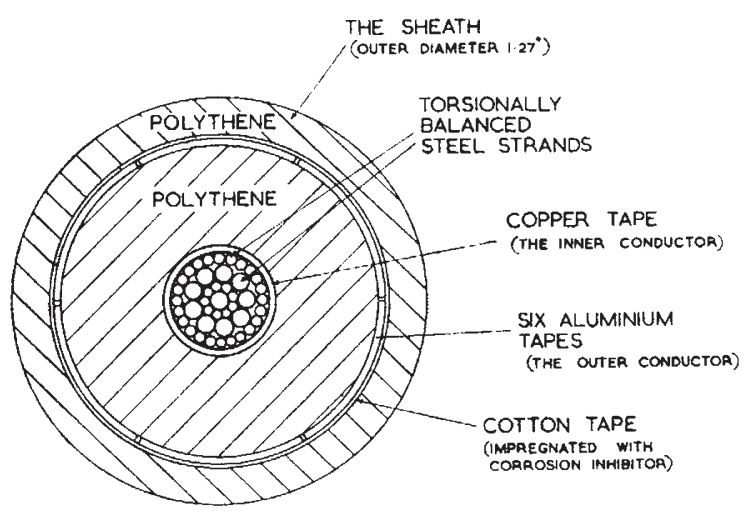

Fig. 1. Cross-gection of proposed light-weight deep-sea callle apparatus for inspecting joints and ylands wer $\Theta$ on show. The Station has long undertaken the supply of thermionic valves for submerged repeaters and has recently completed the design of a new valve. which is, mechanically at least, a big advance on earlier types. The heater, a wire helix of rectangular cross-section and electrically double-ended, fits inside a tube of sintered alumina which in turn fits closely inside the platinum core of the cathode. The design of the other electrodes had to take account of the restricted anode and screen grid voltages imposed by. the need to limit the overall voltage which has to be applied to tho ends of the cable-the ropeaters art fed in series from tho ends-and had to prevent ions formed in the anode/screen grid space from reaching and damaging the cathode. Only very little mica is used in the valve.

Work has continued on techniques and equipment for radio communications using frequencies around 4,000 Mc./s. Frequency control has been obtained by extending conventional multiplication to micro. wave frequencies; the starting point is an oscillator. with quartz resonator, operating at about $50 \mathrm{Mc} . / \mathrm{s}$., and the higher intermediate stages use cavity resonators and triode valves with very close electrode spacings. Long-term stability is better than one part. in $10^{8}$ and short-term better than one part in $10^{8}$. For work involving measurements over the band 3,800-4,200 Mc./s. a method of launching signals, aperiodically, at the short-circuited end of a wave. guide has eliminated all controls except that of the frequency of the test oscillator. The joining together of wave-guides of different rectangular cross-sections has been improved by the use of symmetrically stepped transforming sections. A rapid method of measuring the voltage standing-wave ratio in waveguide, using a rotating loop, was demonstrated ; the ratio can be displayed as a function of frequency over a width of band up to $50 \mathrm{Mc} / \mathrm{s}$. A new modulator, electrically balanced but using only one nonlinear element, has been developed for frequency changing between the intermediate (70 Mc./s.) and micro-wave frequencies.

The Post Office uses correction by artificial echoes on long-distance television links to reduce the lineax wave-form distortions which are not removable by conventional equalization. The standard echo weveform corrector combines the input signal with an adjustable array of advanced and retarded echoes over a range of 3 microseconds. A new corrector uses doublet, or differentiated, echoes in a novel circuit which enables the range to be extended to suit the requirements for individual links. A unit-block construction provides for extensions in steps of 2 microseconds. The cost and size are much less than those of the stiandard model. Fig. 2 show's the response, of a 200-mile cable link, to a pulse (sine-squared in shape and of duration one-third mierosecond at half amplitude) before and after echo correction.

Big savings have been obtained in the United States by converting amplified loaded cablos from four-wire to two-wire working-thereby doubling the traffic handling capacity - following the dovelopment of negative-impedance, valve amplifiers having only 

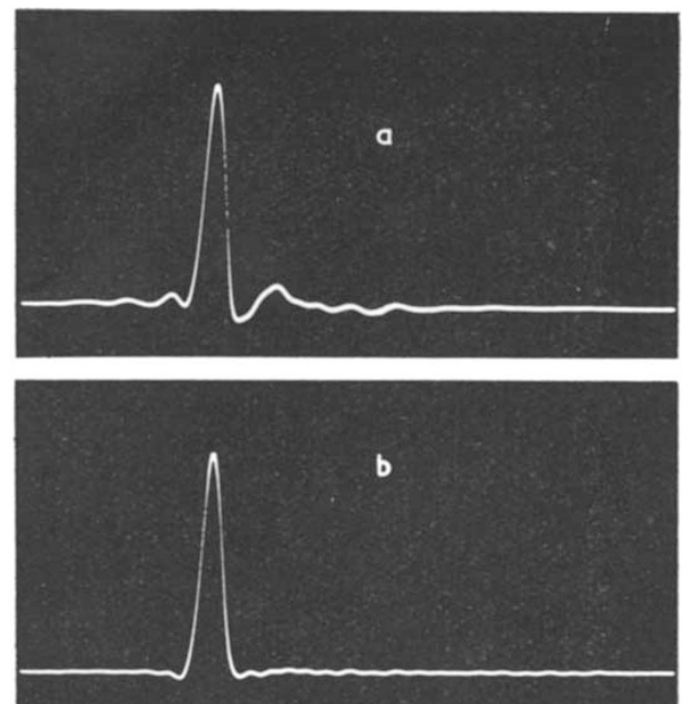
Fig. 2. Response of $200-$ mile cable link to $1 / 3-\mu$ sec. sine-squared

two terminals. But the reduction of attenuation obtained by the insertion of one negative impedor, whether in series or in shunt with the conductors, introduces a serious mismatch and hence loud echoes. If two are inserted at the same point, one in series and one in shunt, and are suitably related to one another and to the cable impedance, echoes can be very much reduced. Such a pair of elements, each using one transistor, have been designed and models are undergoing field trials.

Radio-telegraphy over long distances is being studied in order to minimize the errors in the received messages which arise due to fading, noise and interference. Current practice uses frequency-shift telegraphy, the demodulator in the receiver having a limiter and frequency discriminator; errors arise if signals on either the frequency of the 'space' or that of the 'mark' fade into the noise. The complete message is, however, available on each frequency separately and an improved method of demodulation has been developed which fails only if the signals on both frequencies are simultaneously seriously affected by noise-a rare event because fading is usually frequency selective. Both this study and work on more conventional systems were facilitated by two new equipments, an automatic counter of errors and a fading machine which gives a close approximation to natural fading by combining six signal components in substantially random phase.

Among the larger exhibits was a letter-facing machine, to which attention has been particularly directed since the completion of the design of a lettersorting machine now going into production. The postal mechanization laboratories were showing also a method of classifying information based on the keying of the first few letters of, say, the name of a town.

Some features of speech interpolation were demonstrated on equipment being built to enable technical studies to be made. The idea uses the fact that at no time during a telephone conversation need both a 'go' and a 'return' channel be provided. If a group on $n$ trunks is available, it may sustain $m$ conversations $(m>n)$ without detriment to the con- versations, provided terminal equipment can allocate free one-way trunks to speakers the moment they are needed. A practical equipment may need to be complex, but even so it may be economic if, for example, it can effectively double the capacity of a long oversea trunk route.

A sound spectrograph developed for the study of speech transmission systems based on formant coding is capable of analysing any audio-frequency signals. It indicates on 'Teledeltos' paper the energy contained in the recorded signal being analysed in the frequency band $f-\frac{1}{2} \Delta f$ to $f+\frac{1}{2} \Delta f$, where $\Delta f$ can be selected between 20 and $500 \mathrm{c}$./s. and $f$ is scanned from zero to a maximum value of $5 \mathrm{kc} . / \mathrm{s}$. The level range has been extended, from the $8 \mathrm{db}$. obtainable with the conventional constant-current excitation of the stylus which marks the paper, to $\mathbf{4 0} \mathrm{db}$. by the use of width-modulated pulses of constant amplitude. Among other new apparatus for laboratory use was one for measuring the thickness of tarnish films to within $10 \mathrm{~A}$.

The prize-winning numbers of Premium Savings Bonds are produced by apparatus 'ERNIE' designed at the Station. The key elements, the generators of random digits, are duplicated and their outputs so combined that failure of one does not upset the randomness of the sequences produced. Several electrical and statistical tests are used for checking the randomness. One of the statistical analyses uses some of the conventional tests for randomness, namely, frequency (of individual digits), serial (the frequency of pairs of digits), gap (intervals between successive appearances of the same digit), poker (classification of blocks of four as 'all alike', 'all different', etc.), and coupon collector (size of block containing at least one of each digit). The analysis is carried out automatically on the Pegasus electronic digital computer, the input tapes for the computer being produced automatically (in the Pegasus code) by auxiliary equipment.

A new rolling mill, coupled with the application of powder metallurgy to nickel iron alloys and with closely controlled annealing schedules, has made possible very thin magnetic tapes of consistently high permeability (for example, 40,000). The two work rolls of the four high mill are less than $\frac{1}{2}$ in. in diameter and are supported along their entire lengths; the plane containing their axes is inclined to that containing the axes of the backing rolls. After the final roll, which may be to a thickness as little as 0.0002 in., the tape is slit in another new machine which uses a flexible disk the cutting edge of which is so shaped and deflected as to provide correct cutting clearances.

The Station has of recent years supplied further quartz resonators to many of the leading observatories of the world. A new resonator-a short bar. approximately square in cross-section, with a volume of about 2 c.c. and vibrating torsionally-is under development. It may prove an alternative to the Essen ring, compared with which it is more robust and easier to make; but it has yet to be shown to have quite as high a stability.

Several exhibits dealt with the technology of germanium and components made from it. Sections of junction transistors made by alloying indium to the two faces of a thin wafer showed the accurately plane and parallel junctions obtained when sufficient care is paid to crystal orientation, wetting of the ger. 
manium by the indium, alloying and cooling. The linetics of the gas-phase erosion of germanium are being studied by observations of changes of pressure and weight. Fast junction diodes are being made by heavily doping the germanium with nickel; their performance was demonstrated.

Collectively, the exhibits showed three broad features of progress in telecommunications. First, an increasingly large part is played by electronic engineering, backed by improved materials and new components. Secondly, the role of mechanical engineering is changing as fower new components have moving parts or require fine clearances; it may lie rather in the machines making the components or assembling them. Thirdly, reliability has to be studied more intensively than ever before, and in good time, if applications of new scientific discoveries aro to be successful.

\section{COLOURS AND FLAVOURS}

$\mathrm{T}$

$\mathrm{HE}$ first of a series of four meetings under the general title of "Some Nutritional and Allied Problems confronting the Food Manufacturers", arranged by the Nutrition Panel of the Food Group of the Society of Chemical Industry, was held in the Society's rooms at Belgrave Square, London, on October 23. These meetings are concerned with food additives, the first dealing with "Colours and Flavours" and the subsequent ones with "Antioxidants", "Emulsifying and Dispersing Agents" and "Chemical Preservatives". Interest in food additives has increased considerably since the end of the War, ospecially in view of pending legislation in this field, and the first meeting was very well attended.

The first paper at this meeting, under the chairmanship of Dr. F. Aylward (Borough Polytechnic), was read by Dr. B. R. J. Thomas, of A. Boake, Roberts and Co., Ltd., on the "Technological Aspects of Colourings". Dr. Thomas pointed out that the arguments for the use of colours in food were well known and well founded and were accepted by all authorities. The main object of his talk was to deal with the present British list of permitted colours, indicating the problems it raised for the food colour supplier and the food manufacturer. In view of the importance of appearance in the selection and appreciation of a food, the manufacturer is anxious to obtain the right shade for a particular use to which a colour is put, although public taste can be schooled to accept minor departures from previous standards. From the point of view of cost, colour is not an important item to the manufacturer as long as he has not to change it frequently. Thus, speaking in approximate terms, $1 \mathrm{lb}$. of amaranth will colour 2,000 gallons of raspberryade or 4,000 gallons of ice cream. This means that the cost of colouring raspberryade is approximately $0.09 d$. per gallon or $0.045 d$. per gallon of ice cream, assuming a price of 15s. per lb. for the dye.

Tho desirable properties of an ideal colour were then enumerated. Such a colour must be without suspicion of carcinogenic activity and must be obtainable in a pure form free from carcinogenic impurities. It must also be stable to a temperature range of $-10^{\circ}$ to $100^{\circ} \mathrm{C}$, and also to $\mathrm{pH}$ 's of 2-8 and to light during storage. It should be resistant to oxidation and reduction and to preservatives, especially sulphur dioxide, and there should be no colour variation from batch to batch. The colour should also be obtainable in oil-soluble and watersoluble forms. On the basis of the above properties, the British list ${ }^{1}$ was examined, the discussion being confined mainly to the so-called $A$ colours (colours which, from the available evidence, would seem unlikely to be harmful when consumed in foods in the customary amounts) and vegetable extracts.

Reds. The British list ${ }^{1}$ contains seven red $A$ colours (Ponceau 4R, Carmoisine, Amaranth, Red 6B, Red $F . B .$, Ponceau $S X$ and Fast Red $E$ ) which conform to most of the points mentioned above, although not one of them is oil-soluble and not one of them is absolutely satisfactory from all points of view. Rhodamine $B$, and Erythrosine of seaside rock and canned cherry fame, are now classified as $C$ and $B$ colours, respectively, yet for the specific purposes mentioned they are irreplaceable. ( $B$ colours are those for which the available evidence is deficiont or conflicting although there is no apparent reason on structural or other grounds to expect them to be harmful in the amounts ordinarily consumed. $C$ colours are those which have been shown, or are suspected, to have harmful effects.) Not ono orange colour has been given an $A$ rating, and this means that the manufacturer has to use a blend of other $A$ colours, which at once raises all kinds of difficulties as a result of the differing stabilities of the constituent colours of the blend. The absence of satisfactory red and orange oil-soluble colours is a serious limitation in the coloration of dairy products and frying oils.

Yellows. The three $A$ colours, Tartrazino, Naphthol Yellow $S$ and Sunset Yellow $F^{\prime} . C . F$., cover most of the neods of the colour user, but the alkali stability of these water-soluble dyes is not high enough for all applications. Again, there are no oil-soluble yollows in the $A$ list.

Green and blue. 'The British list' ${ }^{1}$ is very defieient in these colours, two blues only, Indigotin and Blue V.R.S., being available. The former is very unstable, and when green is needed the manufacturer is reduced to the unsatisfactory course of using blends of other dyes. Of blacks, there are no $A$ colours, but Black $B . N$. is on the permitted list as a $B$ colour, although Black B.N., has a definite blue tinge, which is a disadvantage.

After reviewing the British list, Dr. Thomas suggested that an intensive study is needed of higher wave-length colours both from the point of view of use and of carcinogenicity. Furthermore, he pointed out that the British list differed from those of other countries, and this raised difficulties if a food manufacturer wanted to export. Thus, Naphthol Yellow $S$ was on the British list but not on tho American list, while Britain and Australia were the only countries which permitted Blue V.R.S. Light Green S.F. Yellowish was permitted in the majority of countries except Britain. There was therefore a strong argument for some international agreement upon colours permitted as food additives.

A reference was made to vegotable colours which may find inereasing use in the futuro in view of the dearth of synthetic oil-soluble colours. These colours are difficult to produce and standardize. There are ton vegetable extracts in the British list ${ }^{1}$, but apparently only five of those appear in the lists of other countries.

Mr. G. R. A. Short, of W. J. Bush and Co., Ltd., spoke about the "Technological Aspocts of Flavour- 